\title{
Validated surrogate endpoints needed for peri-implantitis
}

\author{
Abstracted from \\ Faggion CM Jr, Listl S, Tu YK. \\ Assessment of endpoints in studies on peri-implantitis treatment: \\ a systematic review. J Dent 2010; 38: 443-450. \\ Address for correspondence: Department of Prosthodontics, \\ Dental School Ruprecht-Karls-University of Heidelberg, Im Neuenheimer Feld 400, \\ 69120 Heidelberg, Germany. E-mail: Clovis.Faggion@med.uni-heidelberg.de
}

\section{Question: Do studies on treatment for peri-implantitis use true endpoints for outcome?}

Data sources Pubmed, Cochrane and Lilac databases, Google, Google Scholar, hand searching of websites of major dental journals. The reference list of five recently published systematic reviews on peri-implantitis treatment were also screened for potential studies. Study selection Randomised controlled trials and non-randomised studies in English, German, French, Spanish and Italian on periimplantitis treatment in humans were included. Case series, case reports and cross sectional or non-therapy studies were excluded from the assessment of endpoints. No minimum follow up time was set for studies that were included.

Data extraction and synthesis Data were extracted in duplicate by two reviewers and disagreements were resolved by consensus. True endpoints for peri-implantitis treatment were considered only if they provided evidence of tangible benefit to the patient. The outcome variables regarded as true endpoints were implant failure, aesthetic assessment and variables related to quality of life, but these were only considered if they were clearly identified as an objective of the research, not as an outcome of treatment. Surrogate endpoints were considered as those measurements of clinical outcomes such as probing pocket depth and clinical attachment level.

Results Fourteen studies were included in this review with data on implant failure presented solely as consequence of peri-implantitis therapy. No true endpoint was described for any study on periimplantitis. Mean pocket probing depth, clinical attachment level and bleeding on probing were the three surrogate endpoints cited most often in the literature.

Conclusions All endpoints used in the trials reviewed are surrogates of clinical events, such as implant failure. Clinical surrogate endpoints should be validated to assess the real effect of these measures on true endpoints.

\section{Commentary}

This systematic review had a clear objective; to address whether previous randomized controlled studies on peri-implantitis therapy use appropriate true endpoints or surrogate endpoints only.

Surrogate endpoints are clinical or lab values that are expected to serve as a reliable substitute for true endpoints. True endpoints in the periodontitis or peri-implantitis treatment would be tooth or implant maintenance or failure. Traditionally used surrogate endpoints on the periodontal therapy were; pocket probing depth, clinical attachment level, bleeding on probing. Sufficient evidence exists to support the view that surrogate endpoints might not reflect true endpoint in the case of chronic disease, ${ }^{1}$ which applies also in case of peri-implantitis. Thus, the review's authors have appropriately addressed narrowly focused questions.

The authors made an extensive search of the literature by using Pubmed, Cochrane and Lilacs. However, they did not mention whether the language was limited or not. Medline and Embase were not used. Grey literature was searched in English, German, French, Spanish and Italian by Google. One possible drawback to this study is that the authors did not present the definition of peri-implantitis in collecting the articles. As peri-implantitis is not a fully defined disease entity yet, it seems to be a necessary measure to define the peri-implantitis in the inclusion/exclusion criteria.

The result of this review indicates that those surrogate endpoints were repeatedly used on the peri-implantitis studies, while true endpoints (implant failures) were reported as a consequence of peri-implantitis therapy, not as an objective of an investigation. The results were not presented as meta-analysis, but the types and the frequency of endpoints were presented. However, meta-analysis in this review would be unnecessary, since it was clearly mentioned that the objective of this review was to report the types and frequency of endpoints usage.

As the authors pointed out, there is clearly a need for more validated surrogate endpoints in lieu of true clinical endpoint in peri-implantitis therapy. However, there is also a concern that, in using 'implant failure' as the only endpoint in the peri-implantitis therapy, this could fail to discriminate between effective therapy for acquiring appropriate mucosal seal around implant and noneffective therapy.

\section{Practice point}

- Traditionally used clinical surrogate endpoints for peri-implantitis treatments are possibly not indicating the true endpoint (implant removal), thus further validation is necessary.

\section{Dong Won Lee}

Department of Periodontology, Gangnam Severance Hospital, College of Dentistry, Yonsei University, Seoul, Korea

1. Fleming TR, DeMets DL. Surrogate end points in clinical trials: are we being misled? Ann Intern Med 1996; 125: 605-613.

Evidence-Based Dentistry (2011) 12, 7. doi:10.1038/sj.ebd.6400770 